\title{
Plane partitions and the combinatorics of some families of reduced Kronecker coefficients.
}

\author{
Laura Colmenarejo $\|^{p}$ \\ ${ }^{1}$ Departamento de Álgebra, Universidad de Sevilla, Sevilla, España
}

\begin{abstract}
We compute the generating function of some families of reduced Kronecker coefficients. We give a combinatorial interpretation for these coefficients in terms of plane partitions. This unexpected relation allows us to check that the saturation hypothesis holds for the reduced Kronecker coefficients of our families. We also compute the quasipolynomial that govern these families, specifying the degree and period. Moving to the setting of Kronecker coefficients, these results imply some observations related to the rate of growth experienced by the families of Kronecker coefficients associated to the reduced Kronecker coefficients already studied.

Résumé. Nous calculons les fonctions génératrices de certaines familles de coefficients de Kronecker réduits. Nous donnons une interprétation combinatoire de ces coefficients à l'aide de partitions planes. Cette relation inattendue nous permet de vérifier que l'hypothèse de saturation est vérifiée pour les coefficients de Kronecker réduits de nos familles. Nous calculons aussi le quasi-polynôme qui gouverne ces familles en spécifiant le degré et la période. En passant au coefficient de Kronecker, ces résultats impliquent certaines observations reliées au taux de croissance des familles de coefficients de Kronecker associés aux coefficients de Kronecker réduits déjà étudiés.
\end{abstract}

Keywords. Combinatorial representation theory, symmetric functions, Kronecker product, combinatorial interpretation of coefficients, stability

\section{Introduction}

Trying to understand the Kronecker coefficients is one of the most important problems in Representation Theory. It has captured the attention of mathematicians for almost a century, but it has remained unsolved. Recently, it has come back to the forefront because of their connections to Geometric Complexity theory, [BMS13, BI08, PP15, BOR09], and to Quantum Information Theory, [CM06, Kly04, Wig37].

The Kronecker coefficients are the multiplicities appearing in the decomposition into irreducible of the tensor product of two irreducible representations of the symmetric group. They also appear naturally in the study of the general lineal group and the unitary group.

In 1938 Murnaghan discovered an intriguing stabilization phenomena for the Kronecker coefficients, [Mur38, Mur56]. They stabilize when we increase the first rows of its three indexing partitions. The limits

$\dagger^{\dagger}$ Email: laurach@us.es. Partially supported by MTM2013-40455-P, P12-FQM-2696, FQM-333, and FEDER. 
of these sequences are known as the reduced Kronecker coefficients. They are indexed by the partitions obtained after deleting the first parts of the original triple.

In [BRR16], Briand, Rattan and Rosas described the effect of increasing the first rows and columns of the partitions indexing a reduced Kronecker coefficient. They found stability for the first situation, and linear growth for the second. Trying to go one step further, we investigate what happen when we add cells to other rows and columns of the partitions indexing reduced Kronecker coefficients. In the present abstract, we study the following families of reduced Kronecker coefficients:

$$
\begin{array}{ll}
\triangleright \text { Family } 1 & \bar{g}_{\left(k^{a}\right),\left(k^{b}\right)}^{(k)} \\
\triangleright \text { Family 2 } & \bar{g}_{\left((k+i)^{a}\right),\left(k^{b}\right)}^{(k)} \\
\triangleright \text { Family 3 } & \bar{g}_{\left(k^{b}\right),\left(k+i, k^{a}\right)}^{(k)}
\end{array}
$$

After a briefly introduction about the reduced Kronecker coefficients and their relation with the Kronecker coefficients, Section 2 focuses on our families of reduced Kronecker coefficients. Definition 2.2 introduces the Kronecker tableaux of C. Ballantine and R. Orellana. They are our main combinatorial tool: in Theorem 2.3 it is shown that they can be used to compute the reduced Kronecker coefficients that we are considering.

The generating function related to Families 1, 2 and 3 are shown in Theorem 2.4. For Family 1, we give the generating function for the reduced Kronecker coefficients $\bar{g}_{\left(k^{a}\right),\left(k^{b}\right)}^{(k)}$. This generating function depends on $a$ and $b$. In the case of Family 2, we show that after some initial zeros, their generating function is exactly the generating function of Family 1 . Then, results related to Family 1 apply also for Family 2. Finally, for Family 3 we give the generating function of the stable value in the diagonals. This phenomenon is explained in Table 3 .

In Section 3 , we give a striking connection to plane partitions. In Theorem 3.2 we show that the reduced Kronecker coefficients corresponding to the different families have a combinatorial description in terms of plane partitions. We obtain this result by comparing the generating function obtained in Theorem 2.4 with MacMahon's classical formula, Theorem 3.1. Plane partitions have appeared before in the study of the Kronecker coefficients of E. Vallejo, [Val00], and L. Manivel, [Man10].

Section 4 is dedicated to some applications. We show that the saturation conjecture of Kirillov and Klyachko holds for these three families of reduced Kronecker coefficients, and that the sequences related to Families 1 and 3 are weakly increasing. In Theorem 4.4 we show that Family 1 and Family 3 are described also by quasipolynomials. We specify the period and the degree of those quasipolynomials.

Finally, we make some observations about the rate of growth experienced by the Kronecker coefficients associated to the families of reduced Kronecker coefficients already studied.

The results concerning Family 1 are joint work with M. Rosas, and were announced in [CR15].

\section{Reduced Kronecker coefficients}

In 1938, Murnaghan introduced the reduced Kronecker coefficients, $\bar{g}_{\alpha \beta}^{\gamma}$, through the Kronecker product, although he did not call them like that. 
Theorem 1.1 (Murnaghan's Theorem, [Mur38] [Mur56]) There exists a family of non-negative integers $\left\{\bar{g}_{\alpha \beta}^{\gamma}\right\}$, indexed by triples of partitions $(\alpha, \beta, \gamma)$, such that, for $\alpha$ and $\beta$ fixed, only finitely many terms $\bar{g}_{\alpha \beta}^{\gamma}$ are non-zero, and for all $n \geq 0$,

$$
s_{\alpha[n]} * s_{\beta[n]}=\sum_{\gamma} \bar{g}_{\alpha \beta}^{\gamma} s_{\gamma[n]},
$$

where $\alpha[n]=\left(n-|\alpha|, \alpha_{1}, \alpha_{2}, \ldots\right)$.

The reduced Kronecker coefficients are directly related to the Kronecker coefficients. Note that $\alpha[n]$ is a partition if and only if $n \geq \alpha_{1}+|\alpha|$. Murnaghan's theorem shows the following stability property for the Kronecker coefficients: for $n$ big enough the expansion of $s_{\alpha[n]} * s_{\beta[n]}$ in the Schur basis does not depend on the first part of the indexing partitions.

In particular, given three partitions $\alpha, \beta$ and $\gamma$, the sequence $\left\{g_{\alpha[n] \beta[n]}^{\gamma[n]}\right\}_{n}$ is eventually constant. The reduced Kronecker coefficient $\bar{g}_{\alpha \beta}^{\gamma}$ can be defined as the stable value of this sequence. Therefore, there exists a positive integer $N$ such that for $n \geq N$

$$
\bar{g}_{\alpha \beta}^{\gamma}=g_{\alpha[n] \beta[n]}^{\gamma[n]} .
$$

The point at which the expansion of the Kronecker product $s_{\alpha[n]} * s_{\beta[n]}$ stabilizes is denoted by $\operatorname{stab}(\alpha, \beta)$. In [BOR11], E. Briand, R. Orellana, and M. Rosas prove that $\operatorname{stab}(\alpha, \beta)=|\alpha|+|\beta|+\alpha_{1}+\beta_{1}$. Like the reduced Kronecker coefficients inherit the symmetry from the Kronecker coefficients, we have the following bound for $N$.

Corollary 1.2 Consider three partitions $\alpha, \beta$ and $\gamma$ such that, for $n \geq N$

$$
\bar{g}_{\alpha \beta}^{\gamma}=g_{\alpha[n] \beta[n]}^{\gamma[n]} .
$$

Then, $N \leq \min \{\operatorname{stab}(\alpha, \beta), \operatorname{stab}(\alpha, \gamma), \operatorname{stab}(\beta, \gamma)\}$.

The reduced Kronecker coefficients are interesting objects of their own right. Littlewood observed that they coincide with the Littlewood-Richardson coefficients when $|\alpha|+|\beta|=|\gamma|$, [Mur55, Lit58]. In fact, it is believed that they are easier to understand than the Kronecker coefficients. Regardless, it has been shown that they contain enough information to compute from them the Kronecker coefficients, [BOR11].

\section{The generating function of several families of reduced Kronecker coefficients}

Before computing the generating functions of these families, we need to introduce the combinatorial tools that we use in our proofs.

Definition 2.1 An $\alpha$-lattice permutation is a sequence of integers such that in every initial part of the sequence the number of occurrences of $i$ plus $\alpha_{i}$ is bigger or equal than the number of occurrences of $i+1$ plus $\alpha_{i+1}$.

Definition 2.2 A Kronecker tableau is a semi-standard Young tableau T of shape $\lambda / \alpha$ and type $\nu / \alpha$, with $\alpha \subset \lambda \cap \nu$, whose reverse reading word is an $\alpha$-lattice permutation, and such that if either $\alpha_{1}=\alpha_{2}$ or $\alpha_{1}>\alpha_{2}$ and any one of the following two conditions is satisfied: 
(i) The number of 1's in the second row of $\lambda / \alpha$ is exactly $\alpha_{1}-\alpha_{2}$.

(ii) The number of 2's in the first row of $\lambda / \alpha$ is exactly $\alpha_{1}-\alpha_{2}$.

We refer to this last condition as $\alpha$-condition. The number of Kronecker tableaux of shape $\lambda / \alpha$ and type $\nu / \alpha$, with $\alpha \subset \lambda \cap \nu$, is denoted by $k_{\alpha \nu}^{\lambda}$.

For instance, consider $\lambda=(5,3,2,1), \nu=(5,4,2)$ and $\alpha=(3,1)$. Then, on the left, there is an example of a tableau that is not a Kronecker tableau, because it does not satisfy the $\alpha$-condition, and on the right, there is an example of a Kronecker tableau:

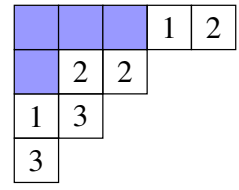

No Kronecker tableau

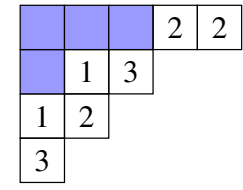

Kronecker tableau

In [BO07], C. Ballantine and R. Orellana introduce the notion of the Kronecker tableaux to give a combinatorial description of a special kind of Kronecker coefficients.

Lemma 2.3 (Theorem 3.2(a), [BO07]) Let $n$ and $p$ be positive integers such that $n \geq 2 p$. Let $\lambda=$ $\left(\lambda_{1}, \ldots, \lambda_{\ell(\lambda)}\right)$ and $\nu$ be partitions of $n$. If $\lambda_{1} \geq 2 p-1$, the multiplicity of $s_{\nu}$ in $s_{(n-p, p)} * s_{\lambda}$ equals $\sum_{\substack{\alpha \vdash p \\ \alpha \subseteq \lambda \cap \nu}} k_{\alpha \nu}^{\lambda}$

The families that we study in this article present different stability properties. Firstly, we present these properties with different examples.

Family 1 has the following stability property for the case $b=a$ : for each fixed $k$, the sequence obtained when $a$ grows stabilizes. Moreover, its generating function depends only on $a$. The following table shows this phenomenon, specifying the references from On-Line Encyclopedia of Integer Sequences.

Tab. 1: Family 1: Case $b=a$, for $a=0, \ldots, 6$

\begin{tabular}{c|cccccccccccccc}
$\mathrm{k}$ & 0 & 1 & 2 & 3 & 4 & 5 & 6 & 7 & 8 & 9 & 10 & 11 & 12 & OEIS \\
\hline $\mathrm{a}=0$ & 1 & 0 & 0 & 0 & 0 & 0 & 0 & 0 & 0 & 0 & 0 & 0 & 0 & $\mathrm{~A} 000007$ \\
$\mathrm{a}=1$ & 1 & 1 & 2 & 2 & 3 & 3 & 4 & 4 & 5 & 5 & 6 & 6 & 7 & $\mathrm{~A} 008619$ \\
$\mathrm{a}=2$ & 1 & 1 & 3 & 4 & 7 & 9 & 14 & 17 & 24 & 29 & 38 & 45 & 57 & $\mathrm{~A} 008763$ \\
$\mathrm{a}=3$ & 1 & 1 & 3 & 5 & 9 & 13 & 22 & 30 & 45 & 61 & 85 & 111 & 150 & $\mathrm{~A} 001993$ \\
$\mathrm{a}=4$ & 1 & 1 & 3 & 5 & 10 & 15 & 26 & 38 & 60 & 85 & 125 & 172 & 243 & $\mathrm{~A} 070557$ \\
$\mathrm{a}=5$ & 1 & 1 & 3 & 5 & 10 & 16 & 28 & 42 & 68 & 100 & 151 & 215 & 312 & $\mathrm{~A} 070558$ \\
$\mathrm{a}=6$ & 1 & 1 & 3 & 5 & 10 & 16 & 29 & 44 & 72 & 108 & 166 & 241 & 357 & $\mathrm{~A} 070559$
\end{tabular}

For $b=a$ in Family $2, \bar{g}_{\left.(k+i)^{a}\right)\left(k^{a}\right)}^{(k)}$, we have that, after some initial zeros, the sequence defined by the non-zero reduced Kronecker coefficients is independent of $i$ and it is equal to the sequence defined by Family 1. Let see this phenomenon with an example. 
Tab. 2: Family 2: case $a=b=2$.

\begin{tabular}{c|ccccccccccccccccc}
$\mathrm{k}$ & 0 & 1 & 2 & 3 & 4 & 5 & 6 & 7 & 8 & 9 & 10 & 11 & 12 & 13 & 14 & 15 & 16 \\
\hline $\mathrm{i}=0$ & 1 & 1 & 3 & 4 & 7 & 9 & 14 & 17 & 24 & 29 & 38 & 45 & 57 & 66 & 81 & 93 & 111 \\
$\mathrm{i}=1$ & 0 & 0 & 0 & 1 & 1 & 3 & 4 & 7 & 9 & 14 & 17 & 24 & 29 & 38 & 45 & 57 & 66 \\
$\mathrm{i}=2$ & 0 & 0 & 0 & 0 & 0 & 0 & 1 & 1 & 3 & 4 & 7 & 9 & 14 & 17 & 24 & 29 & 38 \\
$\mathrm{i}=4$ & 0 & 0 & 0 & 0 & 0 & 0 & 0 & 0 & 0 & 1 & 1 & 3 & 4 & 7 & 9 & 14 & 17 \\
$\mathrm{i}=5$ & 0 & 0 & 0 & 0 & 0 & 0 & 0 & 0 & 0 & 0 & 0 & 0 & 1 & 1 & 3 & 4 & 7
\end{tabular}

We observe that the sequence defined by each row is exactly the third row, when $a=2$, in Table 1

Family 3 shows a stability phenomenon when we look at its diagonals when $b=a+1$. This phenomenon can be seen in the following table for $a=2$ and $b=3$. We look at the sequence formed by the numbers in bold.

Tab. 3: Family 3: case $a=2$ and $b=3$.

\begin{tabular}{c|ccccccccccccccc}
$\mathrm{k}$ & 0 & 1 & 2 & 3 & 4 & 5 & 6 & 7 & 8 & 9 & 10 & 11 & 12 & 13 & OEIS \\
\hline $\mathrm{i}=0$ & $\mathbf{1}$ & 1 & 3 & 4 & 7 & 9 & 14 & 17 & 24 & 29 & 38 & 45 & 57 & 66 & $\mathrm{~A} 008763$ \\
$\mathrm{i}=1$ & 0 & $\mathbf{1}$ & $\mathbf{2}$ & 4 & 7 & 11 & 16 & 23 & 31 & 41 & 53 & 67 & 83 & 102 & $\mathrm{~A} 000601$ \\
$\mathrm{i}=2$ & 0 & 0 & $\mathbf{1}$ & $\mathbf{2}$ & $\mathbf{5}$ & 8 & 14 & 20 & 30 & 40 & 55 & 70 & 91 & 112 & $\mathrm{~A} 006918$ \\
$\mathrm{i}=3$ & 0 & 0 & 0 & $\mathbf{1}$ & $\mathbf{2}$ & $\mathbf{5}$ & $\mathbf{9}$ & 15 & 23 & 34 & 47 & 64 & 84 & 108 & $\mathrm{~A} 014126$ \\
$\mathrm{i}=4$ & 0 & 0 & 0 & 0 & $\mathbf{1}$ & $\mathbf{2}$ & $\mathbf{5}$ & $\mathbf{9}$ & $\mathbf{1 6}$ & 24 & 37 & 51 & 71 & 93 & \\
$\mathrm{i}=5$ & 0 & 0 & 0 & 0 & 0 & $\mathbf{1}$ & $\mathbf{2}$ & $\mathbf{5}$ & $\mathbf{9}$ & $\mathbf{1 6}$ & $\mathbf{2 5}$ & 38 & 54 & 75 & $\mathrm{~A} 175287$ \\
$\mathrm{i}=6$ & 0 & 0 & 0 & 0 & 0 & 0 & $\mathbf{1}$ & $\mathbf{2}$ & $\mathbf{5}$ & $\mathbf{9}$ & $\mathbf{1 6}$ & $\mathbf{2 5}$ & $\mathbf{3 9}$ & 55 &
\end{tabular}

Each diagonal of the table stabilizes to a value of this sequence. We give the generating function for the sequence of the stable values of the diagonals. For instance, in Table 3 the sequence of stable values is $1,2,5,9,16,25,39, \ldots$

Now we are ready to compute the generating function for the families of reduced Kronecker coefficients that we consider.

Theorem 2.4 1. For $b=a$, the generating function of the reduced Kronecker coefficients of Family 1 , $\bar{g}_{\left(k^{a}\right),\left(k^{a}\right)}^{(k)}$, is

$$
\mathcal{F}_{a}=\frac{1}{(1-x)\left(1-x^{2}\right)^{2} \cdots\left(1-x^{a}\right)^{2}\left(1-x^{a+1}\right)} .
$$

2. For $b=a$, the generating function of the reduced Kronecker coefficients of Family 2, $\bar{g}_{\left((k+i)^{a}\right),\left(k^{a}\right)}^{(k)}$, for $k \geq \frac{a(a+1)}{2} \cdot i$ is also $\mathcal{F}_{a}$.

3. For $b=a+1$, the stable value of the $j^{\text {th }}$ diagonal corresponds to the reduced Kronecker coefficients $\bar{g}_{\left(k^{a}\right),\left(2 k-j, k^{a-1}\right)}^{(k)}$, when $k \geq 2 j$. Their generating function is

$$
\mathcal{G}_{a}=\frac{1}{(1-x)^{2}\left(1-x^{2}\right)^{3} \ldots\left(1-x^{a-1}\right)^{3}\left(1-x^{a}\right)^{2}\left(1-x^{a-1}\right)} .
$$


Remark 1 For $a \neq b$ in Families 1 and 2, and for $b \neq a+1$ in Family 3, the reduced Kronecker coefficients take values 0 or 1 .

Proof Sketch of proof:: We present a sketch of the proof for the case corresponding to Family 3.

Fix positive integers $a$ and $j$. The $j^{t h}$ diagonal is described by setting $i=k-j$. We consider the reduced Kronecker coefficients $\bar{g}_{\left(k^{a}\right)\left(2 k-j, k^{a-1}\right)}^{(k)}$. By Corollary 1.2 the reduced Kronecker coefficients can be seen as Kronecker coefficients. Therefore, $\bar{g}_{\left(k^{a}\right)\left(2 k-j, k^{a-1}\right)}^{(k)}$ counts the Kronecker tableaux of shape $\left(3 k, k^{a}\right) / \alpha$ and type $\left(2 k+j, 2 k-j, k^{a-1}\right) / \alpha$, with $\alpha \vdash k$ and $\ell(\alpha) \leq a+1$.

Since $\mathcal{G}_{a}$ is the generating function for coloured partitions with parts in $\mathcal{C}_{a}=\{1, \overline{1}, 2, \overline{2}, \overline{\overline{2}}, \ldots, a-$ $1, \overline{a-1}, \overline{\overline{a-1}}, a, \bar{a}, a+1\}$, it suffices to define a bijection between coloured partitions with parts in $\mathcal{C}_{a}$ and the Kronecker tableaux counted by $\bar{g}_{\left(k^{a}\right)\left(2 k-j, k^{a-1}\right)}^{(k)}$.

We define the bijection with the following algorithm: to a partition $\beta$ of $j$ with parts in $\mathcal{C}_{a}$, we associate a Kronecker tableau $T(\beta)$ as follows. We identify each element of $\mathcal{C}_{a} \cup\{0\}$ with a column of height $a+1$ according to the following scheme:
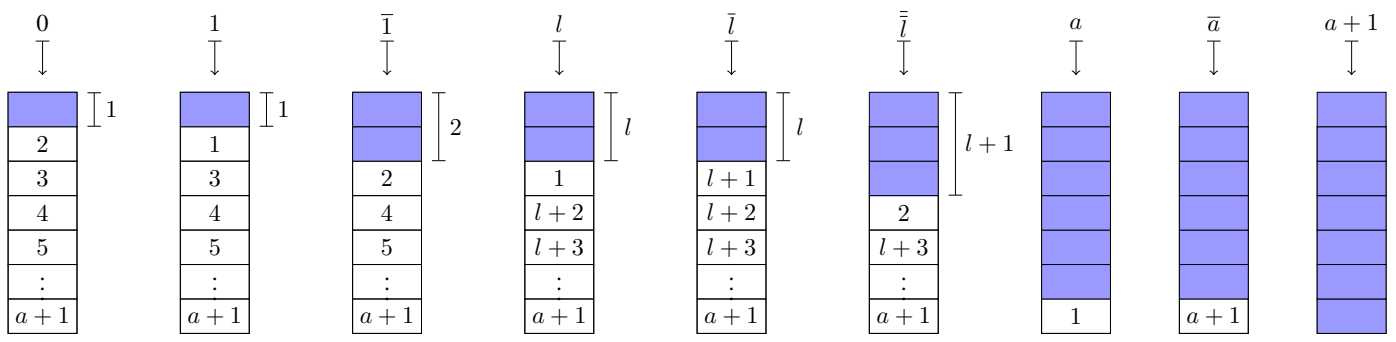

for $l=2, \ldots, a-1$.

Let $m_{i}$, with $i \in \mathcal{C}_{a} \cup\{0\}$, denote the number of times that $i$ appears in $\beta$. Using that $\beta$ is a partition of $j$ and that the blue boxes on these columns form a partition $\alpha$ of $k$, we get that $m_{0}=k-j-m_{\overline{1}}-\sum_{l=2}^{a-1} m_{\overline{\bar{l}}}$. The rest of $m_{i}$ are determined by $\beta$. The partition $\alpha$ of $k$ satisfies the following recursion: $\alpha_{a+1}=m_{a+1}$, $\alpha_{l}=\alpha_{l+1}+m_{i}+m_{\bar{l}}+m_{\overline{\overline{l-1}}}$ for $l=3, \ldots, a, \alpha_{2}=\alpha_{3}+m_{2}+m_{\overline{2}}+m_{\overline{1}}$, and $\alpha_{1}=\alpha_{2}+m_{1}+m_{0}$.

Note that it is always possible to order these columns in such a way that we obtain a semi-standard Young tableau. This semi-standard Young tableau defines the first columns of $T(\beta)$. The rest of the Kronecker tableau of shape $\left(3 k, k^{a}\right) / \alpha$ is built as follows: the $l^{\text {th }}$ row is filled with $l$, for $l=2, \ldots a+1$ and the first row is filled with the remaining numbers of the type $\left(2 k+j, 2 k-j, k^{a-1}\right) / \alpha$ in weakly increasing order from left to right.

For instance, take $a=3, j=3, k=7$ and the coloured partition $\beta=(\overline{\overline{2}}, 1)$. Then, the corresponding Kronecker tableau obtained by our algorithm is

\begin{tabular}{|l|l|l|l|l|l|l|l|l|l|l|l|l|l|l|l|l|l|l|l|l|}
\hline & & & & & 1 & 1 & 1 & 1 & 1 & 1 & 1 & 1 & 1 & 1 & 1 & 2 & 2 & 2 & 2 & 4 \\
\hline & 1 & 2 & 2 & 2 & 2 & 2 & & & & & & & & & & & & & & \\
& 3 & 3 & 3 & 3 & 3 & 3 & & & & & & & & & & & & & \\
\hline & 2 & 4 & 4 & 4 & 4 & 4 & 4 & & & & & & & & & & & & & \\
\hline
\end{tabular}

By the end of this section, we want to underline a study of C. Ballantine and B. Hallahan. In [BH12], they study the stability of the Kronecker product of a Schur function indexed by a hook partition and 
another Schur function indexed by a rectangle partition. They use Blasiak's combinatorial rule, which describes the Kronecker coefficients in terms of Yamanouchi coloured tableaux, [Bla12] as we do with the Kronecker tableaux. They are able to give bounds for the size of the partition for which the Kronecker coefficients are stable and that once the bound is reached, no new Schur functions appear in the decomposition of Kronecker product.

\section{Plane Partitions and Reduced Kronecker Coefficients}

In this section we establish a link between our families of reduced Kronecker coefficients and plane partitions.

A plane partition is a finite subset $\mathcal{P}$ of positive integer lattice points, $\{(i, j, k)\} \subset \mathbb{N}^{3}$, such that if $(r, s, t)$ lies in $\mathcal{P}$ and if $(i, j, k)$ satisfies $1 \leq i \leq r, 1 \leq j \leq s$ and $1 \leq k \leq t$, then $(i, j, k)$ also lies in $\mathcal{P}$. Let $\mathcal{B}(r, s, t)$ be the set of plane partitions fitting in a $r \times s$ rectangle, and with biggest part less than or equal to $t$. As an illustration we present a plane partition in $\mathcal{B}_{4,3,4}$

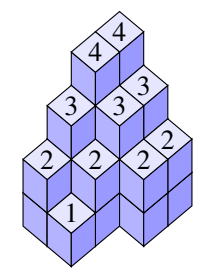

MacMahon showed the generating function for the plane partitions fitting in a rectangle.

Theorem 3.1 (P. MacMahon, [Mac04]) Let $l=\min (r, s)$ and $n=\max (r, s)$. Then, the generating function for the plane partitions fitting inside an $r \times s$ rectangle is

$$
\prod_{j=l}^{n}\left(\frac{1}{1-x^{j}}\right)^{l} \cdot \prod_{i=1}^{l-1}\left(\frac{1}{1-x^{i}}\right)^{i}\left(\frac{1}{1-x^{n+i}}\right)^{l-i} .
$$

Remark 2 MacMahon did not describe the generating function directly like in Theorem 3.1 but few calculations lead to it.

Denote by $\overline{\bar{g}}_{a}(j)=\bar{g}_{\left(k^{a}\right)\left(2 k-j, k^{a-1}\right)}^{(k)}$ with $k \geq 2 j$ the stable value of the $j^{\text {th }}$ diagonal associated to the reduced Kronecker coefficients $\bar{g}_{\left(k+i, k^{a-1}\right),\left(k^{a}\right)}^{(k)}$ appearing in Theorem 2.4 .

Theorem 3.2 1. The reduced Kronecker coefficient of Family $1, \bar{g}_{\left(k^{a}\right),\left(k^{a}\right)}^{(k)}$, counts the number of plane partitions of $k$ fitting inside a $2 \times$ a rectangle.

2. Consider the reduced Kronecker coefficient of Family 2 after shifting the initial zeros $\left\{\bar{g}_{\left((k+(n+1) i)^{a}\right),\left((k+n \cdot i)^{a}\right)}^{(k+n \cdot i)}\right\}_{k, i \geq 0}$, with $n=\frac{a(a+1)}{2}$. It also counts the number of plane partitions of $k$ fitting inside a $2 \times$ a rectangle.

3. We have the following combinatorial interpretation of the reduced Kronecker coefficients $\overline{\bar{g}}_{a}(j)$ :

$$
\overline{\bar{g}}_{a}(j)=\sum_{l=0}^{j} \#\left\{\begin{array}{c}
\text { plane partitions of } l \\
\text { in } 3 \times(a-1) \text { rectangle }
\end{array}\right\} \#\left\{\begin{array}{c}
\text { plane partitions of } j-l \\
\text { in } 2 \times 1 \text { rectangle }
\end{array}\right\} .
$$


Proof Sketch of proof:: The descriptions corresponding to Families 1 and 2 are proved comparing directly the generating function of both combinatorial objects. For Family 3, consider the generating function of the plane partitions fitting inside a $3 \times(a-1)$ rectangle.

$$
\mathcal{H}_{a}=\frac{1}{(1-x)\left(1-x^{2}\right)^{2}\left(1-x^{3}\right)^{3} \ldots\left(1-x^{a-1}\right)^{3}\left(1-x^{a}\right)^{2}\left(1-x^{a+1}\right)} .
$$

By Theorem 2.4 the generating function of the reduced Kronecker coefficients $\overline{\bar{g}}_{a}(j)$ is $\mathcal{G}_{a}$, which is related with $\mathcal{H}_{a}$ by

$$
\mathcal{H}_{a}=(1-x)\left(1-x^{2}\right) \mathcal{G}_{a}
$$

Then, we can express the coefficients appearing in the expansion for $\mathcal{G}_{a}$ in terms of the coefficients of $\mathcal{H}_{a}$. Let $\mathcal{G}_{a}=\sum_{n} q_{n} x^{n}$ be the expansion for $\mathcal{G}_{a}$ and $\mathcal{H}_{a}=\sum_{n} r_{n} x^{n}$ the corresponding for $\mathcal{H}_{a}$. Then, $r_{n}$ is the number of plane partitions fitting inside a $3 \times(a-1)$ rectangle.

Expanding both sides of (1) and equating the coefficients, we obtain the following recursive relation

$$
\begin{aligned}
& r_{0}=q_{0}, \\
& r_{1}=q_{1}-q_{0}, \\
& r_{2}=q_{2}-q_{1}-q_{0}, \\
& r_{n}=q_{n}-q_{n-1}-q_{n-2}+q_{n-3} \quad \text { for all } n \geq 3 .
\end{aligned}
$$

The coefficients $q_{n}$ can be expressed in terms of the $r_{n}$ coefficients.

Lemma 3.3 With the same notation as above,

$$
q_{n}=\sum_{m=0}^{n}\left(\left\lfloor\frac{n-m}{2}\right\rfloor+1\right) r_{m}
$$

The proof of Lemma 3.3 proceeds by induction on $n$. Finally, observe that the coefficients that appears in Lemma 3.3 count the number of plane partitions fitting inside a $2 \times 1$ rectangle.

\section{Applications}

\subsection{Saturation Hypothesis}

Denote by $\left\{C\left(\alpha^{1}, \ldots, \alpha^{n}\right)\right\}$ any family of coefficients depending on the partitions $\alpha^{1}, \ldots, \alpha^{n}$. The family $\left\{C\left(\alpha^{1}, \ldots, \alpha^{n}\right)\right\}$ satisfies the saturation hypothesis if the conditions $C\left(\alpha^{1}, \ldots, \alpha^{n}\right)>0$ and $C\left(s \cdot \alpha^{1}, \ldots, s \cdot \alpha^{n}\right)>0$ for all $s>1$ are equivalent, where $s \cdot \alpha=\left(s \cdot \alpha_{1}, s \cdot \alpha_{2}, \ldots\right)$. The LittlewoodRichardson coefficients satisfy the saturation hypothesis, as was shown by Knutson and Tao in [KT99].

On the other hand, the Kronecker coefficients are known not to satisfy it. For example $g_{(n, n),(n, n)}^{(n, n)}$ is equal to 1 if $n$ is even, and to 0 otherwise, see [BOR09].

In [Kly04] and [Kir04], Kirillov and Klyachko have conjectured that the reduced Kronecker coefficients satisfy the saturation hypothesis. From the combinatorial interpretation for the reduced Kronecker coefficients in terms of plane partitions given in Theorem 3.2, we verify their conjecture for our families of coefficients. 
Corollary 4.1 The saturation hypothesis holds for the coefficients $\bar{g}_{\left(k^{a}\right),\left(k^{a}\right)}^{(k)}$. In fact, $\bar{g}_{\left((s k)^{a}\right),\left((s k)^{a}\right)}^{(s k)}>0$ for all $s \geq 1$. Moreover, the sequences of coefficients obtained by, either fixing $k$ or $a$, and then letting the other parameter grow are weakly increasing.

For Family 2, we have to consider the reduced Kronecker coefficients after the initial zeros. Let $n=$ $\frac{a(a+1)}{2}$.

Corollary 4.2 The saturation hypothesis holds for the coefficients

$$
\bar{g}_{\left((k+(n+1) i)^{a}\right),\left((k+n \cdot i)^{a}\right)}^{(k+n \cdot i)} .
$$

Finally, for Family 3, we have the corresponding result concerning the stable values of the diagonals.

Corollary 4.3 The saturation hypothesis holds for the coefficients $\overline{\bar{g}}_{a}(j)$. Infact, $\overline{\bar{g}}_{a}(s j)>0$ for all $s \geq 1$, where $\overline{\bar{g}}_{a}(s j)$ denotes the associated reduced Kronecker coefficient with its three partitions multiplied by $s$. Moreover, the sequences of coefficients obtained by, either fixing $i$ or a, and then letting $k$ grow, are weakly increasing.

\subsection{Quasipolynomiality}

In Theorem 2.4 we compute the generating functions $\mathcal{F}_{a}$ and $\mathcal{G}_{a}$ for the reduced Kronecker coefficients associated to Families 1 and 3. These are the resulting implications of this calculation.

Theorem 4.4 Let $\mathcal{F}_{a}$ be the generating function for the reduced Kronecker coefficients $\bar{g}_{\left(k^{a}\right),\left(k^{a}\right)}^{(k)}$. Let $\mathcal{G}_{a}$ be the generating function for the reduced Kronecker coefficients $\overline{\bar{g}}_{a}(j)$.

Let $\ell$ be the least common multiple of $1,2, \ldots, a, a+1$.

1. The generating function $\mathcal{F}_{a}$ can be rewritten as $\mathcal{F}_{a}=\frac{P_{a}(x)}{\left(1-x^{\ell}\right)^{2 a}}$, where $P_{a}(x)$ is a product of cyclotomic polynomials. Moreover, $\operatorname{deg}\left(P_{a}(x)\right)=2 a \ell-(a+2) a<2 a \ell-1$.

2. The generating function $\mathcal{G}_{a}$ can be rewritten as $\mathcal{G}_{a}=\frac{Q_{a}(x)}{\left(1-x^{\ell}\right)^{3 a-1}}$, where $Q_{a}(x)$ is a product of cyclotomic polynomials. Moreover $\operatorname{deg}\left(Q_{a}(x)\right)=\ell(3 a-1)-\frac{3}{2}\left(a^{2}+a\right)<\ell(3 a-1)-1$.

3. The coefficients $\bar{g}_{\left(k^{a}\right),\left(k^{a}\right)}^{(k)}$ are described by a quasipolynomial of degree $2 a-1$ and period dividing $\ell$. In fact, we have checked that the period is exactly l for a less than 10.

4. The coefficients $\overline{\bar{g}}_{a}(j)$ are described by a quasipolynomial of degree $3 a-2$ and period dividing $\ell$. In fact, we have checked that the period is exactly l for a less than 7.

Remark 3 Family 2 is included in the results concerning to $\mathcal{F}_{a}$, which is its generating functions after shifting the initial zeros.

\section{Proof Sketch of proof::}

1. We define $P_{a}(x)$ as $P_{a}(x)=\mathcal{F}_{a} \cdot\left(1-x^{l}\right)^{2 a}$. Then, the generating function $\mathcal{F}_{a}$ can be written as in the theorem. Let $\Phi_{i}$ be the $i^{\text {th }}$ cyclotomic polynomial. From the well-known identity $\left(x^{n}-1\right)=$ $\prod_{i \mid n} \Phi_{i}$, we express $\mathcal{F}_{a}$ and $\left(1-x^{l}\right)^{2 a}$ as product of cyclotomic polynomials. The cyclotomic 
polynomials appearing in $\mathcal{F}_{a}$ also appear in $\left(1-x^{l}\right)^{2 a}$, with exponent at least equal to their exponent in $\mathcal{F}_{a}$. Then, $P_{a}$ is a polynomial and it can be written as a product of cyclotomic polynomials. Moreover, $\operatorname{deg}\left(\mathcal{F}_{a}\right)=a(a+2)$, and then, $\operatorname{deg}\left(P_{a}\right)=2 a l-a(a+2)$.

2. We define $Q_{a}(x)$ as $Q_{a}(x)=\mathcal{G}_{a} \cdot\left(1-x^{l}\right)^{3 a-1}$. Then, the generating function $\mathcal{G}_{a}$ can be written as in the theorem. We express $\mathcal{G}_{a}$ and $\left(1-x^{l}\right)^{3 a-1}$ as a product of cyclotomic polynomials, observing that the cyclotomic polynomials appearing in $\mathcal{G}_{a}$ also appear in $\left(1-x^{l}\right)^{3 a-1}$, with at least equal exponent. Then, $Q_{a}$ is a polynomial and it can be written as a product of cyclotomic polynomials. Moreover, $\operatorname{deg}\left(\mathcal{G}_{a}\right)=\frac{3}{2} a(a+1)$, and $\operatorname{deg}\left(Q_{a}\right)=l(a-1)-\frac{3}{2} a(a+1)$.

The other two items follow using Proposition 4.13 of [BS16].

Let see some examples.

Example 1 The coefficients $\bar{g}_{\left(k^{2}\right),\left(k^{2}\right)}^{(k)}$ are given by the quasipolynomial of degree 3 and period 6 .

$$
\bar{g}_{\left(k^{2}\right),\left(k^{2}\right)}^{(k)}=\left\{\begin{array}{lll}
1 / 72 k^{3}+1 / 6 k^{2}+2 / 3 k+1 & \text { if } k \equiv 0 & \bmod 6 \\
1 / 72 k^{3}+1 / 6 k^{2}+13 / 24 k+5 / 18 & \text { if } k \equiv 1 & \bmod 6 \\
1 / 72 k^{3}+1 / 6 k^{2}+2 / 3 k+8 / 9 & \text { if } k \equiv 2 & \bmod 6 \\
1 / 72 k^{3}+1 / 6 k^{2}+13 / 24 k+1 / 2 & \text { if } k \equiv 3 & \bmod 6 \\
1 / 72 k^{3}+1 / 6 k^{2}+2 / 3 k+7 / 9 & \text { if } k \equiv 4 & \bmod 6 \\
1 / 72 k^{3}+1 / 6 k^{2}+13 / 24 k+7 / 18 & \text { if } k \equiv 5 & \bmod 6
\end{array}\right.
$$

These quasipolynomials are computed applying the binomial identity to expand $\left(1-x^{6}\right)^{4}$, and then grouping the monomials in $P_{2}=\Phi_{2}^{2} \Phi_{3}^{3} \Phi_{6}^{4}$ according to their degree mod 6 . For this, we write each number as $n=2 k+r$, with $r \in\{0, \ldots, 5\}$, and rewrite the result in terms of the variable $k$.

Example 2 The coefficients $\overline{\bar{g}}_{2}(j)$ are given by the quasipolynomial of degree 4 and period 6 .

$$
\overline{\bar{g}}_{2}(j)=\left\{\begin{array}{lll}
1 / 288 j^{4}+1 / 16 j^{3}+7 / 18 j^{2}+j+1 & \text { if } j \equiv 0 & \bmod 6 \\
1 / 288 j^{4}+1 / 16 j^{3}+7 / 18 j^{2}+15 / 16 j+175 / 288 & \text { if } j \equiv 1 & \bmod 6 \\
1 / 288 j^{4}+1 / 16 j^{3}+7 / 18 j^{2}+j+8 / 9 & \text { if } j \equiv 2 & \bmod 6 \\
1 / 288 j^{4}+1 / 16 j^{3}+7 / 18 j^{2}+15 / 16 j+23 / 32 & \text { if } j \equiv 3 & \bmod 6 \\
1 / 288 j^{4}+1 / 16 j^{3}+7 / 18 j^{2}+j+8 / 9 & \text { if } j \equiv 4 & \bmod 6 \\
1 / 288 j^{4}+1 / 16 j^{3}+7 / 18 j^{2}+15 / 16 j+175 / 288 & \text { if } j \equiv 5 & \bmod 6
\end{array}\right.
$$

\subsection{On the Kronecker coefficients}

Thanks to the classical stability phenomenon for the Kronecker coefficients discovered by Murnaghan, we can transcribe our results in terms of reduced Kronecker coefficients as results about Kronecker coefficients. These Kronecker coefficients are indexed by partitions whose first parts are large enough. But we observe that the descriptions given in terms of quasipolynomials only depend on $k$ and $a$ for Family 1 , and on $j$ and $a$ for Family 3. This allows us to finish this section with some observations about the rate of growth of the Kronecker coefficients.

Murnaghan observed that the sequences obtained by adding cells to the first parts of the partitions indexing a Kronecker coefficients are eventually constant. In [BRR16], they show that fixed three partitions, the Kronecker coefficients indexed by them stabilize when we increase these partitions with $n$ new boxes 
in their first row and $n$ new boxes in their first column. They also show that the resulting sequence obtained by increasing the sizes of the second rows (keeping the first one very long in comparison) of the partitions indexing the Kronecker coefficients are described by a linear quasipolynomial of period 2.

An interesting question is then to describe what happens when we add cells to arbitrary rows of the partitions indexing a Kronecker (and reduced Kronecker) coefficient. The results presented here and in [CR15] show several cases when we know what happen. For example, for $a=1$, the three families of sequences are described by a linear quasipolynomial of period 2, as is predicted in the work of Briand, Rattan and Rosas, [BRR16]. But when $a=2$, the sequence corresponding to Family 1 is described by a quasipolynomial of degree 3 and the one corresponding to Family 3 is described by a quasipolynomial of degree 4. For $a=3$, the sequences are described by quasipolynomials of degree 5 and degree 7 (respectively), and show on.

Finally, we remark that the sequences described by both families, Families 1 and 3, when we increase the parameter $a$ are weakly increasing, and bounded. These sequences correspond to increase the sizes of the columns.

\section{References}

[BH12] C. Ballantine and B Hallahan. Stability of coefficients in the Kronecker product of a hook and a rectangle. ArXiV: 1412.1431, 2012.

[BI08] P. Bürgisser and C. Ikenmeyer. The complexity of computing Kronecker coefficients. In 20th Annual International Conference on Formal Power Series and Algebraic Combinatorics (FPSAC 2008), Discrete Math. Theor. Comput. Sci. Proc., AJ, pages 357-368. Assoc. Discrete Math. Theor. Comput. Sci., Nancy, 2008.

[Bla12] J. Blasiak. Kronecker coefficients for one hook shape. ArXiV: 1209.2018, 2012.

[BMS13] J. Blasiak, K. D. Mulmuley, and M. Sohoni. Geometric complexity theory. VI: Nonstandard quantum group for the Kronecker problem. available in http://gct.cs.uchicago.edu/gct4.pdf, 2013.

[BO07] C. Ballantine and R. Orellana. A combinatorial interpretation for the coefficients in the Kronecker product $s_{(n-p, p)} * s_{\lambda}$. Sém. Lothar. Combin., 54A:Art. B54Af, 29 pp. (electronic), 2005/07.

[BOR09] E. Briand, R. Orellana, and M. H. Rosas. Reduced Kronecker coefficients and counterexamples to Mulmuley's strong saturation conjecture SH. Comput. Complexity, 18(4):577-600, 2009. With an appendix by Ketan Mulmuley.

[BOR11] E. Briand, R. Orellana, and M. H. Rosas. The stability of the Kronecker product of Schur functions. J. Algebra, 331:11-27, 2011.

[BRR16] E. Briand, A. Rattan, and M. H. Rosas. On the growth of the Kronecker and the reduced Kronecker coefficients. preprint, 2016.

[BS16] M. Beck and R. Sanyal. Combinatorial reciprocity theorems: Enumerative combinatorics with a polyhedral angle. available in http://math.sfsu.edu/beck/papers/crt.pdf, to appear in 2016. 
[CM06] M. Christandl and G. Mitchison. The spectra of quantum states and the Kronecker coefficients of the symmetric group. Comm. Math. Phys., 261(3):789-797, 2006.

[CR15] L. Colmenarejo and M. H. Rosas. Combinatorics on a family of reduced Kronecker coefficients. C. R. Math. Acad. Sci. Paris, 353(10):865-869, 2015.

[Kir04] A. N. Kirillov. An invitation to the generalized saturation conjecture. Publ. Res. Inst. Math. Sci., 40(4):1147-1239, 2004.

[Kly04] A. Klyachko. Quantum marginal problem and representations of the symmetric group. ArXiV: 0409113, 2004.

[KT99] A. Knutson and T. Tao. The honeycomb model of $\mathrm{GL}_{n}(\mathbf{C})$ tensor products. I. Proof of the saturation conjecture. J. Amer. Math. Soc., 12(4):1055-1090, 1999.

[Lit58] D. E. Littlewood. Products and plethysms of characters with orthogonal, symplectic and symmetric groups. Canad. J. Math., 10:17-32, 1958.

[Mac04] P. A. MacMahon. Combinatory analysis. Vol. I, II (bound in one volume). Dover Phoenix Editions. Dover Publications, Inc., Mineola, NY, 2004.

[Man10] L. Manivel. A note on certain Kronecker coefficients. Proc. Amer. Math. Soc., 138(1):1-7, 2010.

[Mur38] F. D. Murnaghan. The Analysis of the Kronecker Product of Irreducible Representations of the Symmetric Group. Amer. J. Math., 60(3):761-784, 1938.

[Mur55] F. D. Murnaghan. On the analysis of the Kronecker product of irreducible representations of $S_{n}$. Proc. Nat. Acad. Sci. U.S.A., 41:515-518, 1955.

[Mur56] F. D. Murnaghan. On the Kronecker product of irreducible representations of the symmetric group. Proc. Nat. Acad. Sci. U.S.A., 42:95-98, 1956.

[PP15] I. Pak and G. Panova. On the complexity of computing Kronecker coefficients. Comput. Complexity, to appear, 2015. ArXiV: 1404.0653.

[Val00] E. Vallejo. Plane partitions and characters of the symmetric group. J. Algebraic Combin., 11(1):79-88, 2000.

[Wig37] E. Wigner. On the consequences of the symmetry of the nuclear hamiltonian on the spectroscopy of nuclei. Phys. Rev., 51:106-119, Jan 1937. 